\title{
Multiple Positive and Nodal Solutions for Semilinear Elliptic Problems with Critical Exponents
}

\section{DaOmin CaO \& Ezzat S. Noussair}

ABSTRACT. We are concerned with the multiplicity of positive and nodal solutions of

$$
-\Delta u=Q(x)|u|^{2^{*}-2} u+\lambda u, \quad x \in \Omega, u \in H_{0}^{1}(\Omega),
$$

where $\Omega$ is a smooth bounded domain of $\mathbb{R}^{N}, 0 \not \equiv Q(x) \geq 0$, $Q \in C(\bar{\Omega})$, and $\lambda \in\left(0, \lambda_{1}\right)$. The existence of $k$ positive solutions (if $N \geq 4$ ) and $k$ nodal solutions (if $N \geq 7$ ) is established for $\lambda \in\left(0, \lambda_{0}\right)$, for some $\lambda_{0}>0$, provided that ${ }_{\bar{\Omega}} \operatorname{Max}$ is achieved as strict local maximums at $k$ interior points in $\Omega$.

1. Introduction. We consider the existence of multiple positive and nodal solutions (solutions which change sign in $\Omega$ ) of the following problem

$$
-\Delta u=Q(x)|u|^{2^{*}-2} u+\lambda u, \quad x \in \Omega, u \in H_{0}^{1}(\Omega),
$$

where $\Omega$ is a smooth bounded domain of $\mathbb{R}^{N}, N \geq 4,2^{*}=2 N /(N-2), \lambda \in$ $\left(0, \lambda_{1}\right)\left(\lambda_{1}\right.$ is the first eigenvalue of $-\Delta$ with zero Dirichlet boundary conditions $)$ and $Q(x) \in C(\bar{\Omega})$ satisfies the following condition:

Condition $(Q) . Q(x) \geq 0$ in $\Omega$ and there exist points $a^{1}, a^{2}, \ldots, a^{k} \in \Omega$ such that $Q\left(a^{j}\right)$ are strict local maximums satisfying

$$
\begin{aligned}
Q\left(a^{j}\right)=Q_{M} & \equiv \underset{\bar{\Omega}}{\operatorname{Max}} Q(x)>0, \\
Q(x)-Q\left(a^{j}\right) & = \begin{cases}O\left(\left|x-a^{j}\right|^{2}\right) & \text { if } N=4, \\
o\left(\left|x-a^{j}\right|^{2}\right) & \text { if } N \geq 5,\end{cases}
\end{aligned}
$$

for $x$ near $a^{j}, j=1,2, \ldots, k$.

Our main results are: 
Theorem 1.1. Suppose condition $(Q)$ holds. Then there exists a positive number $\lambda_{0} \in\left(0, \lambda_{1}\right)$ such that, for $\lambda \in\left(0, \lambda_{0}\right]$, problem (1.1) has at least $k$ positive solutions.

Theorem 1.2. Suppose condition $(Q)$ holds and $N \geq 7$. Then there exists a positive number $\lambda_{0} \in\left(0, \lambda_{1}\right)$ such that, for $\lambda \in\left(0, \lambda_{0}\right]$, problem (1.1) has at least $k$ nodal solutions.

The existence of at least one positive solution of (1.1) has been established for the special case $Q(x)$ is a constant in a remarkable paper by $\mathrm{H}$. Brezis and L. Nirenberg [7], and for $Q(x)$ satisfying condition $(Q)$ at a point $a \in \bar{\Omega}$, by J. F. Escobar [14].

For constant $Q$ and $\Omega$ is a ball, (1.1) has a unique positive solution [20]. The existence of at least one nodal solution of (1.1) was established in [9], [22].

During the past few years the question whether the topology of $\Omega$ or its geometry were responsible for the solvability and/or the multiplicity of positive solutions of problems similar to (1.1) has been the subject of several investigations, beginning from the Pohozaev's classical theorem [19], asserting that (1.1) with $\lambda=0, Q(x) \equiv$ constant has no solution if $\Omega$ is a star shaped domain. Recent important results supporting the topology effect were obtained by, among other people, J. Kazdan and F. Warner [15], J. M. Coron [10], A. Bahri and J. M. Coron [2], asserting that (1.1) with $\lambda=0, Q(x) \equiv 1$ has a positive solution provided $\Omega$ is topologically nontrivial (some homology groups are nontrivial); and results by E. N. Dancer [11], W. Ding [12], D. Passaseo [18], in which contractible domains for which (1.1) has one or more positive solutions have been exhibited. We also mention a recent paper by M. Marchi and F. Pacella [17] in which contractible domains for which (1.1) has nodal solutions are exhibited.

The aim of this paper is to show the effect of the shape of the graph of $Q(x)$ on the existence and multiplicity of both positive and nodal solutions. Our solutions are obtained as local minimum points of the functional

$$
I_{\lambda}(u)=\frac{1}{2} \int_{\Omega}|\nabla u|^{2}-\lambda u^{2}-\frac{1}{2^{*}} \int_{\Omega} Q(x)|u|^{2^{*}},
$$

for $\lambda \in\left(0, \lambda_{1}\right)$, constrained to suitably constructed closed subsets of $H_{0}^{1}(\Omega)$. Our positive solutions will correspond to critical values in $\left(0, S^{N / 2} / N Q_{M}^{(N-2) / 2}\right)$, and our nodal solutions correspond to critical values in $\left(0,2 S^{N / 2} / N Q_{M}^{(N-2) / 2}\right)$. Our results seem to suggest that the geometry of the graph of $Q(x)$ has a similar effect to the geometry of $\Omega$, on the existence and multiplicity of both kinds of solutions. 
Remark 1.3. The hypothesis $N \geq 7$ in Theorem 1.2 is necessary for the existence of radial nodal solutions by a result of F. V. Atkinson, H. Brezis and L. A. Peletier [1].

Remark 1.4. The more general problem

$$
-\Delta u=Q(x)|u|^{2^{*}-2} u+q(x)|u|^{\gamma-1} u
$$

could be considered in a general domain $\Omega \subseteq \mathbb{R}^{N}$, under suitable assumptions on $Q$ and $q$, for $1<\gamma<2^{*}-1$, by similar arguments.

2. Notations and Preliminary Results. Let $\|\cdot\|$ denote the norm of $H_{0}^{1}(\Omega),\|u\|=\int|\nabla u|^{2}$ for all $u \in H_{0}^{1}(\Omega)$. Here all integrals are Lebesgue integrals over $\Omega$ unless otherwise stated.

Let $g: H_{0}^{1}(\Omega) \rightarrow \mathbb{R}^{N}$ be defined by

$$
g(u)=\frac{\int x|u|^{2^{*}}}{\int|u|^{2^{*}}} .
$$

For $r>0, y \in \mathbb{R}^{N}$, set $B_{r}(y)=\left\{x \in \mathbb{R}^{N},|x-y|<r\right\}$ and let $\bar{B}_{r}(y), S_{r}(y)$ denote the closure and the boundary of $B_{r}(y)$, respectively. By condition $(Q)$ we may choose $\ell>0$ small enough so that $B_{2 \ell}\left(a^{j}\right) \subset \Omega$ are disjoint and $Q(x)<$ $Q\left(a^{j}\right)$ for $x \in \bar{B}_{2 \ell}\left(a^{j}\right), x \neq a^{j}, j=1,2, \ldots, k$.

For $\lambda>0, j=1,2, \ldots, k$, define

$$
\Sigma_{\lambda}=\left\{u \in H_{0}^{1}(\Omega): u \not \equiv 0,\left\langle I_{\lambda}^{\prime}(u), u\right\rangle=0\right\}
$$

where $I_{\lambda}^{\prime}(u)$ denotes the Fréchet derivative of $I_{\lambda}(u)$.

For $\lambda>0$ and $j=1,2, \ldots, k$ define

$$
\begin{aligned}
O_{\lambda}^{j} & =\left\{u \in \Sigma_{\lambda}: g(u) \in B_{\ell}\left(a^{j}\right)\right\} \\
U_{\lambda}^{j} & =\left\{u \in \Sigma_{\lambda}: g(u) \in S_{\ell}\left(a^{j}\right)\right\} \\
V_{\lambda}^{j} & =\left\{u: u^{ \pm} \in O_{\lambda}^{j}\right\}, \\
W_{\lambda}^{j}=\left\{u: u^{ \pm} \in \Sigma_{\lambda}, \quad g\left(u^{ \pm}\right) \in \bar{B}_{\ell}\left(a^{j}\right) \text { and either } g\left(u^{+}\right) \in S_{\ell}\left(a^{j}\right)\right. & \\
& \left.\quad \text { or } g\left(u^{-}\right) \in S_{\ell}\left(a^{j}\right)\right\} .
\end{aligned}
$$

None of $O_{\lambda}^{j}, U_{\lambda}^{j}, V_{\lambda}^{j}, W_{\lambda}^{j}$ are empty, as can be easily verified. 
Define

$$
\begin{gathered}
m_{\lambda}^{j}=\inf \left\{I_{\lambda}(u): u \in O_{\lambda}^{j}\right\}, \\
\bar{m}_{\lambda}^{j}=\inf \left\{I_{\lambda}(u): u \in U_{\lambda}^{j}\right\}, \\
M_{\lambda}^{j}=\inf \left\{I_{\lambda}(u): u \in V_{\lambda}^{j}\right\}, \\
\bar{M}_{\lambda}^{j}=\inf \left\{I_{\lambda}(u): u \in W_{\lambda}^{j}\right\}, \\
S=\inf \left\{\int_{\mathbb{R}^{N}}|\nabla u|^{2}: u \in \mathcal{D}_{0}^{1,2}\left(\mathbb{R}^{N}\right), \int_{\mathbb{R}^{N}}|u|^{2^{*}}=1\right\} .
\end{gathered}
$$

In the following lemmas we establish estimates on $m_{\lambda}^{j}, M_{\lambda}^{j}, \bar{M}_{\lambda}^{j}$, which are crucial to our construction of P.S. sequences in the subsets $O_{\lambda}^{j}, V_{\lambda}^{j}$. (The estimate for $\bar{m}_{\lambda}^{j}$ will be given in Section 3.)

Lemma 2.1. For $j=1, \ldots, k$, and $\lambda>0$ we have

$$
m_{\lambda}^{j}<\frac{S^{N / 2}}{N Q_{M}^{(N-2) / 2}} .
$$

Proof. Let $x_{0} \in \mathbb{R}^{N}, \varepsilon>0$, and

$$
U_{\varepsilon, x_{0}}=\frac{C_{N} \varepsilon^{(N-2) / 2}}{\left(\varepsilon^{2}+\left|x-x_{0}\right|^{2}\right)^{(N-2) / 2}}
$$

with $C_{N}=\left(N(N-2) / Q_{M}\right)^{(N-2) / 4}$.

It is easy to verify that $U_{\varepsilon, x_{0}}$ satisfies

$$
-\Delta u=Q_{M}|u|^{2^{*}-2} u \quad \text { in } \mathbb{R}^{N} .
$$

Furthermore, it is well known [7] that the infimum in (2.5) is achieved by the functions $U_{\varepsilon}, x_{0} /\left\|U_{\varepsilon}, x_{0}\right\|_{L^{2 *}\left(\mathbb{R}^{N}\right)}$. by

Let $0<\rho<\ell / 2, \rho$ fixed. Define a radial nonnegative function $\varphi \in C_{0}^{2}\left(\mathbb{R}^{N}\right)$

$$
\varphi(x)= \begin{cases}1 & x \in B_{\rho}(0), \\ 0 & x \notin B_{2 \rho}(0) .\end{cases}
$$

Set $u_{\varepsilon, a^{j}}=U_{\varepsilon, a^{j}} \varphi\left(x-a^{j}\right)$, and we shall simply write $u_{\varepsilon}$ for $u_{\varepsilon, a^{j}}$ when there is no confusion. 
The following estimates can be easily established from similar estimates in [7]:

$$
\begin{aligned}
& \int\left|u_{\varepsilon}\right|^{2^{*}}=K_{2}^{\prime}+O\left(\varepsilon^{N}\right), \\
& \int\left|\nabla u_{\varepsilon}\right|^{2}=K_{1}+O\left(\varepsilon^{N-2}\right), \\
& K_{2}^{\prime}=C_{N}^{2^{*}} \int_{\mathbb{R}^{N}} \frac{d x}{\left(1+|x|^{2}\right)^{N}}=\left\|U_{1,0}\right\|_{L^{2^{*}\left(\mathbb{R}^{N}\right)}}^{2^{*}}, \\
& K_{1}=(N-2)^{2} C_{N}^{2} \int_{\mathbb{R}^{N}} \frac{|x|^{2}}{\left(1+|x|^{2}\right)^{N}} d x=\left\|\left|\nabla U_{1,0}\right|\right\|_{L^{2}\left(\mathbb{R}^{N}\right)}^{2}, \\
& K_{1} / K_{2}=S, \quad K_{2}^{\prime}=K_{2}^{N /(N-2)}, \quad \text { if } N \geq 5, \\
& \int\left|u_{\varepsilon}\right|^{2}=\left\{\begin{array}{l}
K_{3} \varepsilon^{2}+O\left(\varepsilon^{N-2}\right) \\
K_{3} \varepsilon^{2}|\ln \varepsilon|+O\left(\varepsilon^{2}\right) \quad \text { if } N=4, \\
\left(\int\left|u_{\varepsilon}\right|^{2^{*}}\right)^{2 / 2^{*}}=K_{2}+O\left(\varepsilon^{N-2}\right),
\end{array}\right.
\end{aligned}
$$

where $K_{3}$ is a positive constant.

$$
\begin{gathered}
\int\left|u_{\varepsilon}\right| \leq C_{1} \varepsilon^{(N-2) / 2}, \\
\int\left|u_{\varepsilon}\right|^{2^{*}-1} \leq C_{2} \varepsilon^{(N-2) / 2},
\end{gathered}
$$

for some positive constants $C_{1}, C_{2}$, independent of $\varepsilon$.

For $\varepsilon>0$, let $t_{\varepsilon}>0$ be selected such that $v_{\varepsilon} \equiv t_{\varepsilon} u_{\varepsilon} \in \Sigma_{\lambda}$. That is

$$
t_{\varepsilon}^{2^{*}-2}=\frac{\int\left(\left|\nabla u_{\varepsilon}\right|^{2}-\lambda u_{\varepsilon}^{2}\right)}{\int Q(x)\left|u_{\varepsilon}\right|^{2^{*}}} .
$$

Notice that $v_{\varepsilon} \in O_{\lambda}^{j}$. This follows easily from the symmetry of $u_{\varepsilon}$ about $a^{j}$, which implies that $g\left(v_{\varepsilon}\right) \in B_{\ell}\left(a^{j}\right)$. Hence (2.6) will follow if we show that

$$
\sup _{t>0} I_{\lambda}\left(t u_{\varepsilon}\right)<\frac{S^{N / 2}}{N Q_{M}^{(N-2) / 2}} .
$$


To establish (2.14) we set

$$
h(t)=\frac{t^{2}}{2} \int\left(\left|\nabla u_{\varepsilon}\right|^{2}-\lambda u_{\varepsilon}^{2}\right)-\frac{t^{2^{*}}}{2^{*}} \int Q(x) u_{\varepsilon}^{2^{*}},
$$

$h^{\prime}(t)>0$ for $t \in\left(0, t_{\varepsilon}\right), h^{\prime}(t)<0$ for $t>t_{\varepsilon}$, and $h^{\prime}\left(t_{\varepsilon}\right)=0$. Therefore,

$$
\begin{aligned}
\sup _{t>0} I_{\lambda}\left(t u_{\varepsilon}\right) & =I_{\lambda}\left(t_{\varepsilon} u_{\varepsilon}\right) \\
& =\frac{t_{\varepsilon}^{2}}{N} \int\left|\nabla u_{\varepsilon}\right|^{2}-\lambda u_{\varepsilon}^{2} \\
& =\frac{1}{N}\left[\left(K_{1}+O\left(\varepsilon^{N-2}\right)\right)-K_{3} \eta(\varepsilon) \lambda\right] t_{\varepsilon}^{2},
\end{aligned}
$$

where

$$
\eta(\varepsilon)= \begin{cases}\varepsilon^{2} & \text { if } N \geq 5 \\ \varepsilon^{2}|\ln \varepsilon| & \text { if } N=4 .\end{cases}
$$

Using condition $(Q)$ we have

$$
\begin{aligned}
\int Q(x) u_{\varepsilon}^{2^{*}} & =\int Q_{M}\left|u_{\varepsilon}\right|^{2^{*}}+\int\left(Q(x)-Q_{M}\right)\left|u_{\varepsilon}\right|^{2^{*}} \\
& = \begin{cases}Q_{M} K_{2}^{\prime}+O\left(\varepsilon^{N}\right)+o\left(\varepsilon^{2}\right) & \text { if } N \geq 5 \\
Q_{M} K_{2}^{\prime}+O\left(\varepsilon^{N}\right)+O\left(\varepsilon^{2}\right) & \text { if } N=4\end{cases}
\end{aligned}
$$

Thus

$$
t_{\varepsilon}^{2}=\left[\frac{K_{1}+O\left(\varepsilon^{N-2}\right)-\lambda K_{3} \eta(\varepsilon)}{Q_{M} K_{2}^{\prime}+O\left(\varepsilon^{N}\right)+\alpha(\varepsilon)}\right]^{(N-2) / 2},
$$

where

$$
\alpha(\varepsilon)= \begin{cases}o\left(\varepsilon^{2}\right) & \text { if } N \geq 5 \\ O\left(\varepsilon^{2}\right) & \text { if } N=4\end{cases}
$$

Using (2.16) we have

$$
\sup _{t>0} I_{\lambda}\left(t u_{\varepsilon}\right)=\frac{K_{1}^{N / 2}}{N Q_{M}^{(N-2) / 2}\left(K_{2}^{\prime}\right)^{(N-2) / 2}} \beta(\varepsilon)=\frac{S^{N / 2}}{N Q_{M}^{(N-2) / 2}} \beta(\varepsilon),
$$

where

$$
\beta(\varepsilon)= \begin{cases}1-\frac{N}{2} \frac{K_{3}}{K_{1}} \lambda \varepsilon^{2}+o\left(\varepsilon^{2}\right) & \text { if } N \geq 5, \\ 1-2 \frac{K_{3}}{K_{1}} \lambda \varepsilon^{2}|\ln \varepsilon|+O\left(\varepsilon^{2}\right) & \text { if } N=4 .\end{cases}
$$

It then follows that $\beta(\varepsilon)<1$ for sufficiently small $\varepsilon$, which implies $(2.14)$, and Lemma 2.1 follows. 
Lemma 2.2. Assume condition $(Q)$ holds. Then there exist $\varepsilon>0$ and $\lambda_{\varepsilon}>0$ such that

$$
\text { (i) } \quad \bar{m}_{\ell}^{j}>\frac{S^{N / 2}}{N Q_{M}^{(N-2) / 2}}+\varepsilon,
$$

(ii) $\bar{M}_{\lambda}^{j}>\frac{2 S^{N / 2}}{N Q_{M}^{(N-2) / 2}}+\varepsilon$,

for $j=1,2, \ldots, k$, and $\lambda \in\left(0, \lambda_{\varepsilon}\right)$.

Proof. To prove (i) suppose to the contrary that we could find a sequence $\lambda_{n} \rightarrow 0$ as $n \rightarrow \infty$, such that $\bar{m}_{\lambda_{n}}^{j} \rightarrow c \leq S^{N / 2} / N Q_{M}^{(N-2) / 2}$. Consequently, there exists $u_{n} \in U_{\lambda_{n}}^{j}$ such that

$$
\begin{aligned}
I_{\lambda_{n}}\left(u_{n}\right) & \rightarrow c, \\
\int\left|\nabla u_{n}\right|^{2}-\lambda_{n} u_{n}^{2} & =\int Q(x)\left|u_{n}\right|^{2^{*}} .
\end{aligned}
$$

It then follows easily that $\left\{u_{n}\right\}$ is bounded in $H_{0}^{1}(\Omega)$, and, in particular, $\lambda_{n} \int u_{n}^{2} \rightarrow 0$ as $n \rightarrow \infty$. By Hölder's and Sobolev's inequalities we can fix $\nu>0$ such that

$$
\int\left|\nabla u_{n}\right|^{2}, \int Q(x)\left|u_{n}\right|^{2^{*}} \geq \nu>0
$$

for all $n=1,2, \ldots$. Therefore, we may choose $t_{n}>0$ so that $v_{n}=t_{n} u_{n}$ satisfies

$$
\int\left|\nabla v_{n}\right|^{2}=\int Q_{M}\left|v_{n}\right|^{2^{*}}
$$

and

$$
t_{n}=\left(\frac{\int Q(x)\left|u_{n}\right|^{2^{*}}+\lambda_{n} \int u_{n}^{2}}{\int Q_{M}\left|u_{n}\right|^{2^{*}}}\right)^{(N-2) / 4}
$$

are bounded. Suppose $t_{n} \rightarrow t_{0}$ as $n \rightarrow \infty$. Then $t_{0} \leq 1$ since $Q(x) \leq Q_{M}$ and $\lambda_{n} \int u_{n}^{2} \rightarrow 0$ as $n \rightarrow \infty$. In fact $t_{0}=1$. This follows easily from

$$
\begin{aligned}
\frac{S^{N / 2}}{N Q_{M}^{(N-2) / 2}} & \leq \lim _{n \rightarrow \infty} \frac{1}{N} \int\left|\nabla v_{n}\right|^{2}=\lim _{n \rightarrow \infty} \frac{t_{n}^{2}}{N} \int\left|\nabla u_{n}\right|^{2} \\
& =\lim _{n \rightarrow \infty} t_{n}^{2} \frac{1}{N} \int\left(\left|\nabla u_{n}\right|^{2}-\lambda_{n} u_{n}^{2}\right) \\
& =\lim _{n \rightarrow \infty} t_{n}^{2} I_{\lambda_{n}}\left(u_{n}\right)=t_{0}^{2} c \\
& \leq t_{0}^{2} \frac{S^{N / 2}}{N Q_{M}^{(N-2) / 2}} .
\end{aligned}
$$


The inequalities above also show that

$$
c=\frac{S^{N / 2}}{N Q_{M}^{(N-2) / 2}} \quad \text { and } \quad \lim _{n \rightarrow \infty} \int\left|\nabla v_{n}\right|^{2}=\frac{S^{N / 2}}{Q_{M}^{(N-2) / 2}} .
$$

Set $w_{n}=v_{n} /\left(\int\left|v_{n}\right|^{2^{*}}\right)^{1 / 2^{*}}$. It is easy to verify that

$$
\int\left|\nabla w_{n}\right|^{2} \rightarrow S, \quad \text { as } n \rightarrow \infty
$$

That is, $\left\{w_{n}\right\}$ is a minimizing sequence for the problem

$$
S=\inf \left\{\int|\nabla u|^{2}: u \in H_{0}^{1}(\Omega), \int|u|^{2^{*}}=1\right\}
$$

We now use a result of $\mathrm{P}$. L. Lions [16] to conclude that we can find a point $x_{0} \in \bar{\Omega}$ and a subsequence, still denoted by $\left\{w_{n}\right\}$, such that

$$
\lim _{n \rightarrow \infty} \int_{\Omega} v\left|w_{n}\right|^{2^{*}}=v\left(x_{0}\right)
$$

for any $v \in C(\bar{\Omega})$. In particular, we have

$$
g^{i}\left(u_{n}\right)=\frac{\int x_{i}\left|u_{n}\right|^{2^{*}}}{\int\left|u_{n}\right|^{2^{*}}}=\frac{\int x_{i}\left|w_{n}\right|^{2^{*}}}{\int\left|w_{n}\right|^{2^{*}}} \rightarrow\left(x_{0}\right)_{i},
$$

as $n \rightarrow \infty$, and since $g\left(u_{n}\right) \in S_{\ell}\left(a^{j}\right), x_{0} \in S_{\ell}\left(a^{j}\right)$. Using (2.19) and (2.20) we also have

$$
\begin{aligned}
\lim _{n \rightarrow \infty} \int Q(x)\left|u_{n}\right|^{2} & =\lim _{n \rightarrow \infty} \int Q(x)\left|v_{n}\right|^{2^{*}} \\
& =\frac{Q\left(x_{0}\right)}{Q_{M}} \lim _{n \rightarrow \infty} \int Q_{M}\left|v_{n}\right|^{2^{*}} \\
& =\frac{Q\left(x_{0}\right)}{Q_{M}} \lim _{n \rightarrow \infty} \int\left|\nabla v_{n}\right|^{2} \\
& =\frac{Q\left(x_{0}\right)}{Q_{M}} \frac{S^{N}}{Q_{M}^{(N-2) / 2}},
\end{aligned}
$$




$$
\begin{aligned}
\lim _{n \rightarrow \infty} I_{\lambda_{n}}\left(u_{n}\right) & =\frac{1}{N} \lim _{n \rightarrow \infty} \int Q(x)\left|u_{n}\right|^{2^{*}} \\
& =\frac{1}{N} \lim _{n \rightarrow \infty} \int Q(x)\left|v_{n}\right|^{2^{*}} \\
& =\frac{Q\left(x_{0}\right)}{N Q_{M}} \lim _{n \rightarrow \infty} \int Q_{M}\left|v_{n}\right|^{2^{*}} \\
& =\frac{Q\left(x_{0}\right)}{Q_{M}} \frac{S^{N / 2}}{N Q_{M}^{(N-2) / 2}}<\frac{S^{N / 2}}{N Q_{M}^{(N-2) / 2}},
\end{aligned}
$$

contradicting (2.19). Hence (i) follows.

The proof of (ii) is similar to the proof above. We argue, as before, by way of contradiction. If, to the contrary, we have a sequence $\lambda_{n} \rightarrow 0$ as $n \rightarrow \infty$, and $u_{n} \in W_{\lambda_{n}}^{j}$ satisfying

$$
\begin{gathered}
I_{\lambda_{n}}\left(u_{n}\right) \rightarrow \bar{C} \leq \frac{2 S^{N / 2}}{N Q_{M}^{(N-2) / 2}}, \\
\int\left|\nabla u_{n}^{ \pm}\right|^{2}-\lambda_{n}\left|u_{n}^{ \pm}\right|^{2}=\int Q(x)\left|u_{n}^{ \pm}\right|^{2^{*}},
\end{gathered}
$$

then

$$
\begin{aligned}
I_{\lambda_{n}}\left(u_{n}^{+}\right) & \rightarrow C_{1} \\
I_{\lambda_{n}}\left(u_{n}^{-}\right) & \rightarrow C_{2}, \\
C_{1}+C_{2} & =\bar{C}, \quad \text { and } \\
C_{1} \text { or } C_{2} & \leq \frac{S^{N / 2}}{N Q_{M}^{(N-2) / 2}} .
\end{aligned}
$$

As in the proof of part (i), we can show that $C_{1}$ and $C_{2}=S^{N / 2} / N Q_{M}^{(N-2) / 2}$, which leads to a contradiction, as in the proof of part (i).

Lemma 2.3. Assume condition $(Q)$ holds and $\lambda \in\left(0, \lambda_{1}\right)$. Then

(i) Any sequence $\left\{u_{n}\right\} \subset \Sigma_{\lambda}$ satisfying

$$
\begin{aligned}
& I_{\lambda}\left(u_{n}\right) \rightarrow c<\frac{S^{N / 2}}{N Q_{M}^{(N-2) / 2},} \\
& I_{\lambda}^{\prime}\left(u_{n}\right) \rightarrow 0, \quad \text { as } n \rightarrow \infty,
\end{aligned}
$$

is relatively compact in $H_{0}^{1}(\Omega)$. 
(ii) Any sequence $\left(v_{n}\right) \subset V_{\lambda}^{j}$ satisfying

$$
\begin{aligned}
& I_{\lambda}\left(v_{n}\right) \rightarrow c<m_{\lambda}^{j}+\frac{S^{N / 2}}{N Q_{M}^{(N-2) / 2}}, \\
& I_{\lambda}^{\prime}\left(v_{n}\right) \rightarrow 0, \quad \text { as } n \rightarrow \infty,
\end{aligned}
$$

is relatively compact in $H_{0}^{1}(\Omega)$.

Proof. (i): Since $\lambda \in\left(0, \lambda_{1}\right)$, it is easy to show that $\left\{u_{n}\right\}$ is bounded in $H_{0}^{1}(\Omega)$ and, therefore, we may assume that for some $u_{0} \in H_{0}^{1}(\Omega)$,

$$
\begin{gathered}
u_{n} \rightarrow u_{0} \text { weakly in } H_{0}^{1}(\Omega), \\
u_{n} \rightarrow u_{0} \text { a.e. in } \Omega, \\
\int\left|\nabla u_{0}\right|^{2}-\lambda u_{0}^{2}=\int Q(x)\left|u_{0}\right|^{2^{*},} \\
I_{\lambda}\left(u_{0}\right)=\frac{1}{N} \int Q(x)\left|u_{0}\right|^{2^{*}}>0 \text { if } u_{0} \not \equiv 0 .
\end{gathered}
$$

Let $w_{n}=u_{n}-u_{0}$. By Brezis-Lieb Lemma [5] we have

$$
\begin{aligned}
\int Q(x)\left|u_{n}\right|^{2^{*}} & =\int Q(x)\left|u_{0}\right|^{2^{*}}+\int Q(x)\left|w_{n}\right|^{2^{*}}+o(1), \\
\int\left|\nabla u_{n}\right|^{2} & =\int\left|\nabla u_{0}\right|^{2}+\int\left|\nabla w_{n}\right|^{2}+o(1) .
\end{aligned}
$$

If $\left\|w_{n}\right\| \rightarrow 0$ we are done, so assume $\left\|w_{n}\right\| \rightarrow L>0$. It follows from

$$
\begin{aligned}
\int\left|\nabla w_{n}\right|^{2} & =\int\left|\nabla u_{n}\right|^{2}-\int\left|\nabla u_{0}\right|^{2}+o(1) \\
& =\lambda \int\left|u_{n}\right|^{2}+\int Q(x)\left|u_{n}\right|^{2^{*}}-\int\left|\nabla u_{0}\right|^{2}+o(1) \\
& =\lambda \int\left|u_{n}\right|^{2}+\int Q(x)\left|w_{n}\right|^{2^{*}}+\int Q(x)\left|u_{0}\right|^{2^{*}}-\int\left|\nabla u_{0}\right|^{2}+o(1) \\
& =\int Q(x)\left|w_{n}\right|^{2^{*}}+o(1),
\end{aligned}
$$

that we can choose $t_{n}>0$ so that

$$
\int\left|\nabla t_{n} w_{n}\right|^{2}=\int Q_{M}\left|t_{n} w_{n}\right|^{2^{*}}
$$


In fact,

$$
t_{n}^{4 /(N-2)}=\frac{\int Q(x)\left|w_{n}\right|^{2^{*}}+o(1)}{\int Q_{M}\left|w_{n}\right|^{2^{*}}},
$$

and $t_{n} \rightarrow t_{0} \leq 1$. Therefore,

$$
\begin{aligned}
\frac{1}{N} \lim _{n \rightarrow \infty} \int\left|\nabla w_{n}\right|^{2} & \geq \frac{t_{0}^{2}}{N} \lim _{n \rightarrow \infty} \int\left|\nabla w_{n}\right|^{2} \\
& =\frac{1}{N} \lim _{n \rightarrow \infty} \int\left|\nabla t_{n} w_{n}\right|^{2} \\
& \geq \frac{S^{N / 2}}{N Q_{M}^{(N-2) / N}}
\end{aligned}
$$

where the last inequality follows from the definition of $S$. We then have

$$
\lim _{n \rightarrow \infty} \int\left|\nabla w_{n}\right|^{2} \geq \frac{S^{N / 2}}{Q_{M}^{(N-2) / 2}},
$$

and therefore,

$$
\begin{aligned}
\lim _{n \rightarrow \infty} I_{\lambda}\left(u_{n}\right) & =I_{\lambda}\left(u_{0}\right)+\lim _{n \rightarrow \infty} I_{\lambda}\left(w_{n}\right) \\
& =I_{\lambda}\left(u_{0}\right)+\frac{1}{N} \lim _{n \rightarrow \infty} \int\left|\nabla w_{n}\right|^{2} \\
& \geq I_{\lambda}\left(u_{0}\right)+\frac{S^{N / 2}}{N Q_{M}^{(N-2) / 2}} \\
& \geq \frac{S^{N / 2}}{N Q_{M}^{(N-2) / 2}},
\end{aligned}
$$

contradicting the hypothesis in (i). Therefore $\left\|w_{n}\right\| \rightarrow L>0$ is impossible, and (i) follows.

(ii): Since $\left\{v_{n}\right\} \subset V_{\lambda}^{j}$, we have $v_{n}^{ \pm} \in O_{\lambda}^{j}$ and $I_{\lambda}\left(v_{n}^{ \pm}\right) \geq m_{\lambda}^{j}$. We also have

$$
\begin{gathered}
\int\left|\nabla v_{n}^{ \pm}\right|^{2}-\lambda\left|v_{n}^{ \pm}\right|^{2}=\int Q(x)\left|v_{n}^{ \pm}\right|^{2^{*}}, \\
I_{\lambda}\left(v_{n}\right)=I_{\lambda}\left(v_{n}^{+}\right)+I_{\lambda}\left(v_{n}^{-}\right) .
\end{gathered}
$$


Since $I_{\lambda}\left(v_{n}\right) \rightarrow c<m_{\lambda}^{j}+S^{N / 2} / N Q_{M}^{(N-2) / 2}$, and $I_{\lambda}\left(v_{n}^{ \pm}\right) \geq m_{\lambda}^{j}$, as $n \rightarrow \infty$, we must have

$$
\lim _{n \rightarrow \infty} I_{\lambda}\left(v_{n}^{ \pm}\right)<\frac{S^{N / 2}}{N Q_{M}^{(N-2) / 2}}
$$

It is easy to show that $\left\{v_{n}\right\}$ is bounded in $H_{0}^{1}(\Omega)$, and we may assume that

$$
\begin{aligned}
& v_{n} \rightarrow v_{0} \text { weakly in } H_{0}^{1}(\Omega), \\
& v_{n} \rightarrow v_{0} \text { a.e. in } \Omega, \\
& v_{n}^{+} \rightarrow v_{0}^{+} \text {weakly in } H_{0}^{1}(\Omega), \\
& v_{n}^{-} \rightarrow v_{0}^{-} \text {weakly in } H_{0}^{1}(\Omega) .
\end{aligned}
$$

Let $w_{n}^{ \pm}=v_{n}^{ \pm}-v_{0}^{ \pm}$. If $\left\|w_{n}^{ \pm}\right\| \rightarrow L^{ \pm}>0$, we establish the contradiction

$$
\lim _{n \rightarrow \infty} I_{\lambda}\left(v_{n}^{ \pm}\right) \geq \frac{S^{N / 2}}{N Q_{M}^{(N-2) / 2}}
$$

as in part (i). Hence $\left\|w_{n}\right\| \rightarrow 0$, as $n \rightarrow \infty$, which implies that $v_{n} \rightarrow v_{0}$ strongly in $H_{0}^{1}(\Omega)$. Thus Lemma 2.3 is proved.

Lemma 2.4. Assume condition $(Q)$ holds. Then there exists a $\lambda_{0} \in\left(0, \lambda_{1}\right)$ and a sequence $\left\{u_{n}^{j}\right\} \subset O_{\lambda}^{j}$, for each $j=1,2, \ldots, k$, satisfying $u_{n} \geq 0$,

$$
\begin{aligned}
& I_{\lambda}\left(u_{n}^{j}\right) \rightarrow m_{\lambda}^{j}, \\
& I_{\lambda}^{\prime}\left(u_{n}^{j}\right) \rightarrow 0,
\end{aligned}
$$

as $n \rightarrow \infty$, for $\lambda \in\left(0, \lambda_{0}\right]$.

Proof. We first notice that for $\lambda<\lambda_{1}$ there is a positive constant $\delta=$ $\delta\left(\lambda_{1}-\lambda\right)$ such that $\|u\|_{L^{2^{*}}(\Omega)} \geq \delta>0$ for all $u \in O_{\lambda}^{j}$. Therefore, $\bar{O}_{\lambda}^{j}=O_{\lambda}^{j} \cup U_{\lambda}^{j}$ and $U_{\lambda}^{j}$ is the boundary of $\bar{O}_{\lambda}^{j}$ for $\lambda<\lambda_{1}$ and each $j=1,2, \ldots, k$.

Using Lemmas 2.1 and 2.2 we see that there exists $\lambda_{0} \in\left(0, \lambda_{1}\right)$ such that

$$
m_{\lambda}^{j}<\bar{m}_{\lambda}^{j}
$$

for $\lambda \in\left(0, \lambda_{0}\right], j=1, \ldots, k$. It follows that

$$
m_{\lambda}^{j}=\inf \left\{I_{\lambda}(u): u \in \bar{O}_{\lambda}^{j}\right\}
$$


Fix $\lambda \in\left(0, \lambda_{0}\right]$, and let $\left\{u_{n}^{j}\right\} \subset \bar{O}_{\lambda}^{j}$ be a minimizing sequence for (2.23). By replacing $u_{n}^{j}$ with $\left|u_{n}^{j}\right|$, if necessary, we may assume that $u_{n}^{j} \geq 0$. By applying Ekeland's variational principle [13] we construct a minimizing sequence $\left\{v_{n}\right\} \subset$ $\bar{O}_{\lambda}^{j}$, for each $j=1,2, \ldots, k$, with the properties

(a) $I_{\lambda}\left(v_{n}\right) \leq I_{\lambda}\left(u_{n}^{j}\right)<m_{\lambda}^{j}+\frac{1}{n}$,

(b) $\left\|v_{n}-u_{n}^{j}\right\| \leq \frac{1}{n}$

(c) $I_{\lambda}\left(v_{n}\right)<I_{\lambda}(w)+\frac{1}{n}\left\|w-v_{n}\right\|$ for each $w \neq v_{n}$ in $\bar{O}_{\lambda}^{j}$.

Using (2.22) we may assume that $v_{n} \in O_{\lambda}^{j}$ for sufficiently large $n$. We may now employ the argument in [21] to construct, for each $v_{n}$, an $\varepsilon_{n}>0$ and a functional $t^{n}(w)$ defined for $w \in H_{0}^{1}(\Omega),\|w\| \leq \varepsilon_{n}$ such that $t^{n}(w)\left(v_{n}-w\right) \in O_{\lambda}^{j}$, and

$$
\left\langle\left(t^{n}\right)^{\prime}(0), v\right\rangle=\frac{2 \int\left(\nabla v_{n} \cdot \nabla v-\lambda v_{n} v\right)-2^{*} \int Q(x)\left|v_{n}\right|^{2^{*}-2} v_{n} v}{\int\left|\nabla v_{n}\right|^{2}-\lambda v_{n}^{2}-\left(2^{*}-1\right) \int Q(x)\left|v_{n}\right|^{2^{*}}} .
$$

Choose $0<\delta<\varepsilon_{n}$. Let $0 \not \equiv u \in H_{0}^{1}(\Omega)$ and let $\omega_{\delta}=\delta u /\|u\|$. Fix $n$ and let $z_{\delta}=t^{n}\left(w_{\delta}\right)\left(v_{n}-w_{\delta}\right)$. Since $z_{\delta} \in O_{\lambda}^{j}$ by the properties of $t^{n}\left(w_{\delta}\right)$,

$$
I_{\lambda}\left(z_{\delta}\right)-I_{\lambda}\left(v_{n}\right) \geq-\frac{1}{n}\left\|z_{\delta}-v_{n}\right\|
$$

follows from (2.24). The mean value theorem then gives

$$
\left\langle I_{\lambda}^{\prime}\left(v_{n}\right), z_{\delta}-v_{n}\right\rangle+o\left(\left\|z_{\delta}-v_{n}\right\|\right) \geq-\frac{1}{n}\left\|z_{\delta}-v_{n}\right\| .
$$

Hence

$$
\left\langle I_{\lambda}^{\prime}\left(v_{n}\right),\left(v_{n}-w_{\delta}\right)+\left(t^{n}\left(w_{\delta}\right)-1\right)\left(v_{n}-w_{\delta}\right)-v_{n}\right\rangle \geq-\frac{1}{n}\left\|z_{\delta}-v_{n}\right\|+o\left(\left\|z_{\delta}-v_{n}\right\|\right),
$$

which implies that

$$
\begin{aligned}
& -\left\langle I_{\lambda}^{\prime}\left(v_{n}\right), w_{\delta}\right\rangle+\left(t^{n}\left(w_{\delta}\right)-1\right)\left\langle I_{\lambda}^{\prime}\left(v_{n}\right), v_{n}-w_{\delta}\right\rangle \\
\geq & -\frac{1}{n}\left\|z_{\delta}-v_{n}\right\|+o\left(\left\|z_{\delta}-v_{n}\right\|\right) .
\end{aligned}
$$


Since $t^{n}\left(w_{\delta}\right)\left(v_{n}-w_{\delta}\right) \in O_{\lambda}^{j},\left\langle I_{\lambda}^{\prime}\left(z_{\delta}\right)\right.$,

$$
\left.t^{n}\left(w_{\delta}\right)\left(v_{n}-w_{\delta}\right)\right\rangle=0 .
$$

Thus it follows from (2.26) that

$$
\begin{gathered}
-\delta\left\langle I_{\lambda}^{\prime}\left(v_{n}\right), \frac{u}{\|u\|}\right\rangle+\frac{t^{n}\left(w_{\delta}\right)-1}{t^{n}\left(w_{\delta}\right)}\left\langle I_{\lambda}^{\prime}\left(z_{\delta}\right), t^{n}\left(w_{\delta}\right)\left(u_{n}-w_{\delta}\right)\right\rangle \\
+\left(t^{n}\left(w_{\delta}\right)-1\right)\left\langle I_{\lambda}^{\prime}\left(v_{n}\right)-I_{\lambda}^{\prime}\left(z_{\delta}\right), v_{n}-w_{\delta}\right\rangle \\
\geq-\frac{1}{n}\left\|z_{\delta}-v_{n}\right\|+o\left(\left\|z_{\delta}-v_{n}\right\|\right) .
\end{gathered}
$$

Hence

$$
\begin{aligned}
\left\langle I_{\lambda}^{\prime}\left(v_{n}\right), \frac{u}{\|u\|}\right\rangle \leq \frac{1}{n} \frac{\left\|z_{\delta}-v_{n}\right\|}{\delta}+\frac{o\left(\left\|z_{\delta}-v_{n}\right\|\right)}{\delta} & \\
& +\frac{\left(t^{n}\left(w_{\delta}\right)-1\right)}{\delta}\left\langle I_{\lambda}^{\prime}\left(v_{n}\right)-I_{\lambda}^{\prime}\left(z_{\delta}\right), u_{n}-w_{\delta}\right\rangle .
\end{aligned}
$$

But $\left\|z_{\delta}-v_{n}\right\| \leq \delta+\left|t^{n}\left(w_{\delta}\right)-1\right| C$,

$$
\lim _{\delta \rightarrow 0} \frac{\left|t^{n}\left(w_{\delta}\right)-1\right|}{\delta} \leq\left\|t^{n}(0)\right\| \leq C
$$

for some constant $C>0$, independent of $\delta$, as can be easily verified from (2.25). For fixed $n$, letting $\delta \rightarrow 0$ in (2.27), we obtain

$$
\left\langle I_{\lambda}^{\prime}\left(v_{n}\right), \frac{u}{\|u\|}\right\rangle \leq \frac{C}{n},
$$

which implies that $I_{\lambda}^{\prime}\left(v_{n}\right) \rightarrow 0$, as $n \rightarrow \infty$, and by $(2.24)$ (b) we conclude that $I_{\lambda}^{\prime}\left(u_{n}^{j}\right) \rightarrow 0$ for $\lambda \in\left(0, \lambda_{0}\right)$. This completes the proof of Lemma 2.4 .

\section{Existence of Positive and Nodal Solutions.}

Proof of Theorem 1.1. By combining Lemmas 2.3 and 2.4 we see that there exists $\lambda_{0}>0$ such that for $\lambda \in\left(0, \lambda_{0}\right], j=1,2, \ldots, k$, we have a minimizing sequence $\left\{u_{n}^{j}\right\} \subset O_{\lambda}^{j}$ such that

$$
\begin{aligned}
& u_{n}^{j} \geq 0, \\
& I_{\lambda}\left(u_{n}^{j}\right) \rightarrow m_{\lambda}^{j}, \\
& I_{\lambda}^{\prime}\left(u_{n}^{j}\right) \rightarrow 0, \\
& u_{n}^{j} \rightarrow u^{j} \text { strongly in } H_{0}^{1}(\Omega) .
\end{aligned}
$$

It then follows that $u^{j} \not \equiv 0$ is a weak solution of (1.1) and $u^{j} \geq 0$. By standard regularity argument and the strong maximum principle, we obtain $u^{j}(x)>0$ in $\Omega$, and since $g\left(u^{j}\right) \in B_{\ell}\left(a^{j}\right)$ and $B_{\ell}\left(a^{j}\right)$ are disjoint for $j=1,2, \ldots, k$, we conclude that $u^{j}, j=1, \ldots, k$, are distinct positive solutions of (1.1). This completes the proof of Theorem 1.1. 
The next two lemmas are needed for the proof of Theorem 1.2.

Lemma 3.1. Suppose condition $(Q)$ holds and $N \geq 7$. Then

$$
M_{\lambda}^{j}<m_{\lambda}^{j}+\frac{S^{N / 2}}{N Q_{M}^{(N-2) / 2}}
$$

for $\lambda \in\left(0, \lambda_{1}\right)$.

Proof. The inequality (3.1) follows from the definition of $M_{\lambda}^{j}$ if we show that:

(i) For each $\lambda \in\left(0, \lambda_{1}\right)$ and $\varepsilon>0$ sufficiently small, there exist $\alpha_{\varepsilon}, \beta_{\varepsilon} \in\left[\frac{1}{2}, 2\right]$ such that $\left(\alpha_{\varepsilon} u^{j}-\beta_{\varepsilon} u_{\varepsilon}\right)^{ \pm} \in O_{\lambda}^{j}$, where $u^{j}$, for each $j=1,2, \ldots, k$, is the positive solution constructed in Theorem 1.1, and

$$
u_{\varepsilon} \equiv u_{\varepsilon, a^{j}}=\frac{C_{N} \varepsilon^{(N-2) / 2} \varphi\left(x-a^{j}\right)}{\left(\varepsilon^{2}+\left|x-a^{j}\right|^{2}\right)^{(N-2) / 2}}
$$

where $\varphi$ is a radial nonnegative function, $\varphi \in C_{0}^{2}\left(\mathbb{R}^{N}\right)$, and

$$
\varphi(x)= \begin{cases}1 & x \in B_{\rho}(0) \\ 0 & x \notin B_{2 \rho}(0)\end{cases}
$$

where $\rho$ is a fixed number such that $0<\rho<\ell / 2$.

(ii)

$$
\sup _{\alpha, \beta \in[1 / 2,2]} I_{\lambda}\left(\alpha u^{j}-\beta u_{\varepsilon}\right)<m_{\lambda}^{j}+\frac{S^{N / 2}}{N Q_{M}^{(N-2) / 2}}
$$

Proof of (i).

Set

$$
\begin{aligned}
d & =\min \left\{u^{j}(x):\left|x-a^{j}\right| \leq 2 \rho\right\}, \\
d^{\prime} & =\max \left\{u^{j}(x):\left|x-a^{j}\right| \leq 2 \rho\right\}, \\
A & =2\left(\frac{4 C_{N}}{d}\right)^{1 /(N-2)}, \\
B & =\frac{1}{2}\left(\frac{C_{N}}{4 d^{\prime}}\right)^{1 / N-2} .
\end{aligned}
$$


It is easy to check, using the definition of $u_{\varepsilon, a^{j}}$, that $\left|x-a^{j}\right| \geq \sqrt{\varepsilon} A$ implies that $\frac{1}{2} u^{j}(x) \geq 2 u_{\varepsilon}(x)$; and $\left|x-a^{j}\right| \leq \sqrt{\varepsilon} B$ implies that $2 u^{j}(x) \leq \frac{1}{2} u_{\varepsilon}(x)$. Now set

$$
\begin{aligned}
& \Omega_{\varepsilon}^{+}=\left\{x \in \Omega: \alpha u^{j}(x) \geq \beta u_{\varepsilon}(x)\right\} \\
& \Omega_{\varepsilon}^{2}=\left\{x \in \Omega:\left|x-a^{j}\right| \geq \sqrt{\varepsilon} A\right\} \\
& \Omega_{\varepsilon}^{3}=\left\{x \in \Omega:\left|x-a^{j}\right| \geq \sqrt{\varepsilon} B\right\} .
\end{aligned}
$$

It then follows that

$$
\Omega_{\varepsilon}^{2} \subset \Omega_{\varepsilon}^{+} \subset \Omega_{\varepsilon}^{3}
$$

Next we estimate

$$
\left.\int_{\Omega} Q(x)\left|\left(\alpha u^{j}-\beta u_{\varepsilon}\right)^{ \pm}\right|^{2^{*}}, \quad \int_{\Omega}\left|\left(\alpha u^{j}-\beta u_{\varepsilon}\right)^{ \pm}\right|^{2}, \quad \int_{\Omega} \mid \nabla\left(\alpha u^{j}-\beta u_{\varepsilon}\right)^{ \pm}\right)\left.\right|^{2} .
$$

We have

$$
\begin{aligned}
& \int_{\Omega} Q(x)\left|\left(\alpha u^{j}-\beta u_{\varepsilon}\right)^{+}\right|^{2^{*}} \\
= & \int_{\Omega_{\varepsilon}^{+}} Q(x)\left(\alpha u^{j}-\beta u_{\varepsilon}\right)^{2^{*}} \\
\geq & \int_{\Omega_{\varepsilon}^{2}} Q(x)\left(\alpha u^{j}-\beta u_{\varepsilon}\right)^{2^{*}} \\
\geq & \int_{\Omega_{\varepsilon}^{2}} Q(x)\left(\alpha u^{j}\right)^{2^{*}}-2^{*} \alpha^{2^{*}-1} \beta \int_{\Omega_{\varepsilon}^{2}} Q(x)\left|u^{j}\right|^{2^{*}-1} u_{\varepsilon} \\
\geq & \int_{\Omega} Q(x)\left|\alpha u^{j}\right|^{2^{*}}-\int_{\Omega \backslash \Omega_{\varepsilon}^{2}} Q(x)\left|\alpha u^{j}\right|^{2^{*}}-2^{*} \alpha^{2^{*}-1} \beta \int_{\Omega_{\varepsilon}^{2}} Q(x)\left|u^{j}\right|^{2^{*}-1} u_{\varepsilon},
\end{aligned}
$$

where we used the inequality $(a-b)^{2^{*}} \geq a^{2^{*}}-2^{*} a^{2^{*}-1} b$, for $a \geq b$.

We also have

$$
\begin{aligned}
\int_{\Omega} Q(x)\left|\left(\alpha u^{j}-\beta u_{\varepsilon}\right)^{+}\right|^{2^{*}} & \leq \int_{\Omega} Q(x)\left|\alpha u^{j}\right|^{2^{*}} \\
\int_{\Omega \backslash \Omega_{\varepsilon}^{2}} Q(x)\left|\alpha u^{j}\right|^{2^{*}} & \leq C \varepsilon^{N / 2} \\
\int_{\Omega_{\varepsilon}^{2}} Q(x)\left|u^{j}\right|^{2^{*}-1} u_{\varepsilon} & \leq C_{1} \varepsilon^{(N-2) / 2}
\end{aligned}
$$


for some positive constants $C, C_{1}$, depending only on $Q_{M},\left\|u^{j}\right\|_{L^{\infty}(\Omega)}$, as can be easily verified. From the inequalities above we see that

$$
\left.\left|\int_{\Omega} Q(x)\right|\left(\alpha u^{j}-\beta u_{\varepsilon}\right)^{+}\right|^{2^{*}}-\int_{\Omega} Q(x)\left|\alpha u^{j}\right|^{2^{*}} \mid \leq O\left(\varepsilon^{(N-2) / 2}\right) .
$$

Similarly we have

$$
\begin{aligned}
& \int_{\Omega}\left|\nabla\left(\alpha u^{j}-\beta u_{\varepsilon}\right)^{+}\right|^{2}=\int_{\Omega_{\varepsilon}^{+}}\left|\nabla\left(\alpha u^{j}-\beta u_{\varepsilon}\right)^{+}\right|^{2} \\
& \geq \int_{\Omega_{\varepsilon}^{+}}\left|\nabla\left(\alpha u^{j}\right)\right|^{2}-2 \alpha \beta \int_{\Omega_{\varepsilon}^{+}}\left|\nabla u^{j}\right|\left|\nabla u_{\varepsilon}\right| \\
& \geq \int_{\Omega}\left|\nabla\left(\alpha u^{j}\right)\right|^{2}-\int_{\Omega \mid \Omega_{\varepsilon}^{+}}\left|\nabla\left(\alpha u^{j}\right)\right|^{2} \\
& \geq \int_{\Omega}\left|\nabla\left(\alpha u^{j}\right)\right|^{2}-4|| \nabla u^{j} \|_{L^{\infty}(\Omega)} \omega_{N} \varepsilon^{N / 2} \\
& \int_{\Omega} \mid \nabla\left(\left.\alpha u^{j}\right|^{2}\right)^{1 / 2}\left(\int_{\Omega_{\varepsilon}^{+}}\left|\nabla u_{\varepsilon}\right|^{2}\right)^{1 / 2} \\
&\left.-8 u_{\varepsilon}\right)\left.^{+}\right|^{2} \leq \int_{\Omega_{\varepsilon}^{3}}\left|\nabla\left(\alpha u^{j}-\beta u_{\varepsilon}\right)^{+}\right|^{2} \\
&\left.\leq \int_{\Omega_{\varepsilon}^{3}}\left|\nabla\left(\alpha u^{j}\right)\right|^{2}+\int_{\Omega_{\varepsilon}^{3}} \beta^{2}\left|\nabla u_{\varepsilon}\right|^{2}+8 \int_{\Omega_{\varepsilon}^{+}}\left|\nabla u_{\varepsilon}\right|^{2}\right)^{1 / 2} \\
& \leq \int_{\Omega}\left|\nabla\left(\alpha u^{j}\right)\right|^{2}+4 \int_{\left|x-a^{j}\right| \geq \sqrt{\varepsilon} B}\left|\nabla u_{\varepsilon}\right|^{2} \\
&+8 u_{\varepsilon} \mid \\
& \\
&+\mid \nabla u^{j} \|_{L^{2}(\Omega)}\left(\int_{\left|x-a^{j}\right| \geq \sqrt{\varepsilon} B}\left|\nabla u_{\varepsilon}\right|^{2}\right)^{1 / 2}
\end{aligned}
$$

Easy calculations shows that $\int_{\left|x-a^{j}\right| \geq \sqrt{\varepsilon} B}\left|\nabla u_{\varepsilon}\right|^{2} \leq C \varepsilon^{N / 2}$ for some positive constant $C$, depending only on $\left\|\nabla u^{j}\right\|_{L^{\infty}(\Omega)}$.

The inequalities above then imply that

$$
\left.\left|\int_{\Omega}\right| \nabla\left(\alpha u^{j}-\beta u_{\varepsilon}\right)^{+}\right|^{2}-\int_{\Omega}\left|\nabla\left(\alpha u^{j}\right)\right|^{2} \mid \leq C \varepsilon^{N / 4}
$$


for some constant $C$, depending only on $Q, Q_{M},\left\|\nabla u^{j}\right\|_{L^{\infty}(\Omega)}$.

Similar calculations show that

$$
\left.\left|\int_{\Omega}\right|\left(\alpha u^{j}-\beta u_{\varepsilon}\right)^{+}\right|^{2}-\int_{\Omega}\left|\alpha u^{j}\right|^{2} \mid \leq C \varepsilon^{N / 4} .
$$

Define:

$$
\begin{gathered}
h^{+}(\alpha, \beta)=\int_{\Omega}\left|\nabla\left(\alpha u^{j}-\beta u_{\varepsilon}\right)^{+}\right|^{2}-\lambda\left|\left(\alpha u^{j}-\beta u_{\varepsilon}\right)^{+}\right|^{2} \\
-\int_{\Omega} Q(x)\left|\left(\alpha u^{j}-\beta u_{\varepsilon}\right)^{+}\right|^{2^{*}} .
\end{gathered}
$$

Since $u^{j} \in O_{\lambda}^{j}, \int_{\Omega}\left|\nabla u^{j}\right|^{2}-\lambda \int_{\Omega}\left|u^{j}\right|^{2}-\int_{\Omega} Q(x)\left|u^{j}\right|^{2^{*}}=0$, it then follows from (3.3), (3.4), (3.5) and (3.6) that

$$
h^{+}(\alpha, \beta)=\int_{\Omega}\left|\nabla \alpha u^{j}\right|^{2}-\lambda\left|\alpha u^{j}\right|^{2}-\int_{\Omega} Q(x)\left|\alpha u^{j}\right|^{2^{*}}+O\left(\varepsilon^{N / 4}\right),
$$

and therefore, for small $\varepsilon>0$ we have

$$
h^{+}\left(\frac{1}{2}, \beta\right)>0, \quad h^{+}(2, \beta)<0, \quad \text { for any } \beta \in\left[\frac{1}{2}, 2\right] .
$$

Now define:

$$
\begin{gathered}
h^{-}(\alpha, \beta)=\int_{\Omega}\left|\nabla\left(\alpha u^{j}-\beta u_{\varepsilon}\right)^{-}\right|^{2}-\lambda\left|\left(\alpha u^{j}-\beta u_{\varepsilon}\right)^{-}\right|^{2} \\
-\int_{\Omega} Q(x)\left|\left(\alpha u^{j}-\beta u_{\varepsilon}\right)^{-}\right|^{2^{*}} .
\end{gathered}
$$

Then

$$
h^{-}(\alpha, \beta)=\int_{\Omega}\left|\nabla\left(\beta u_{\varepsilon}-\alpha u^{j}\right)^{+}\right|^{2}-\lambda\left|\left(\beta u_{\varepsilon}-\alpha u^{j}\right)^{+}\right|^{2}-\int_{\Omega} Q(x)\left|\left(\beta u_{\varepsilon}-\alpha u^{j}\right)^{+}\right|^{2^{*}} .
$$

Set $\Omega_{\varepsilon}^{-}=\left\{x \in \Omega: \beta u_{\varepsilon}>\alpha u^{j}\right\}$, so $\Omega_{\varepsilon}^{-}=\Omega \backslash \Omega_{\varepsilon}^{+}$, which implies that $\Omega \backslash \Omega_{\varepsilon}^{3} \subset$ $\Omega_{\varepsilon}^{-} \subset \Omega \backslash \Omega_{\varepsilon}^{2}$.

Using similar calculations to the above, we obtain

$$
\begin{aligned}
\left.\left|\int_{\Omega} Q(x)\right|\left(\beta u_{\varepsilon}-\alpha u^{j}\right)^{+}\right|^{2^{*}}-\int_{\Omega} Q(x)\left|\beta u_{\varepsilon}\right|^{2^{*}} \mid & \leq C \varepsilon^{(N-2) / 4}, \\
\left.\left|\int_{\Omega}\right| \nabla\left(\beta u_{\varepsilon}-\alpha u^{j}\right)^{+}\right|^{2}-\int_{\Omega}\left|\nabla \beta u_{\varepsilon}\right|^{2} \mid & \leq C \varepsilon^{N / 4}, \\
\left.\left|\int_{\Omega}\right|\left(\beta u_{\varepsilon}-\alpha u^{j}\right)^{+}\right|^{2}-\int_{\Omega}\left|\beta u_{\varepsilon}\right|^{2} \mid & \leq C \varepsilon^{N / 4},
\end{aligned}
$$


and

$$
\begin{aligned}
h^{-}(\alpha, \beta) & =\int_{\Omega}\left|\nabla\left(\beta u_{\varepsilon}\right)\right|^{2}-\lambda \int\left(\beta u_{\varepsilon}\right)^{2}-\int_{\Omega} Q(x)\left(\beta u_{\varepsilon}\right)^{2^{*}}+O\left(\varepsilon^{(N-2) / 4}\right) \\
& =\beta^{2} \int_{\Omega}\left|\nabla u_{\varepsilon}\right|^{2}-\lambda \beta^{2} \int\left|u_{\varepsilon}\right|^{2}-\beta^{2^{*}} \int_{\Omega} Q(x)\left(u_{\varepsilon}\right)^{2^{*}}+O\left(\varepsilon^{(N-2) / 4}\right) .
\end{aligned}
$$

We now use (3.10) and the estimates in (2.7), (2.8), (2.9) to obtain (here $U_{1,0}$ is as in (2.7))

$$
\begin{gathered}
h^{-}(\alpha, \beta)=\beta^{2}\left\|\left|\nabla U_{1,0}\right|\right\|_{L^{2}\left(R^{N}\right)}^{2}-\beta^{2^{*}} Q_{M}\left\|U_{1,0}\right\|_{L^{2}\left(\mathbb{R}^{N}\right)}^{2^{*}}+O\left(\varepsilon^{N-2}\right) \\
-\lambda K_{3} \varepsilon^{2}+O\left(\varepsilon^{(N-2) / 4}\right),
\end{gathered}
$$

and since $\left\|\left|\nabla U_{1,0}\right|\right\|_{2}^{2}=Q_{M}\left\|U_{1,0}\right\|_{2^{*}}^{2^{*}},(3.11)$ implies that, for $\varepsilon$ sufficiently small,

$$
h^{-}\left(\alpha, \frac{1}{2}\right)>0, \quad h^{-}(\alpha, 2)<0, \quad \text { for any } \alpha \in\left[\frac{1}{2}, 2\right] .
$$

From (3.8), (3.12), and a theorem by Miranda [18], we conclude that there exist $\alpha_{\varepsilon}, \beta_{\varepsilon} \in\left[\frac{1}{2}, 2\right]$ such that

$$
h^{+}\left(\alpha_{\varepsilon}, \beta_{\varepsilon}\right)=h^{-}\left(\alpha_{\varepsilon}, \beta_{\varepsilon}\right)=0,
$$

which means that $\left(\alpha_{\varepsilon} u^{j}-\beta_{\varepsilon} u_{\varepsilon}\right)^{ \pm} \in \Sigma_{\lambda}$.

To complete the proof of (i) we show next that

$$
g\left(\left(\alpha_{\varepsilon} u^{j}-\beta_{\varepsilon} u_{\varepsilon}\right)^{ \pm}\right) \in B_{\ell}\left(a^{j}\right),
$$

for small $\varepsilon$.

We consider $g\left(\left(\alpha_{\varepsilon} u^{j}-\beta_{\varepsilon} u_{\varepsilon}\right)^{+}\right)$first. We have

$$
\left|\left(\alpha_{\varepsilon} u^{j}-\beta_{\varepsilon} u_{\varepsilon}\right)^{+}\right|^{2^{*}} \leq\left|\alpha_{\varepsilon} u^{j}\right|^{2^{*}},
$$

and

$$
\left|\left(\alpha_{\varepsilon} u^{j}-\beta_{\varepsilon} u_{\varepsilon}\right)^{+}\right|^{2^{*}} \geq\left|\alpha_{\varepsilon} u^{j}\right|^{2^{*}}-2^{*} \alpha_{\varepsilon}^{2^{*}-1} \beta_{\varepsilon}\left|u^{j}\right|^{2^{*}-1} u_{\varepsilon},
$$

on $\Omega_{\varepsilon}^{+}$. Therefore,

$$
0 \geq\left|\left(\alpha_{\varepsilon} u^{j}-\beta u_{\varepsilon}\right)^{+}\right|^{2^{*}}-\left|\alpha u^{j}\right|^{2^{*}} \geq-2^{*} \alpha_{\varepsilon}^{2^{*}-1} \beta_{\varepsilon}\left|u^{j}\right|^{2^{*}-1} u_{\varepsilon},
$$

and for any $x_{i}$ we have

$$
\left|\int_{\Omega} x_{i}\left[\left|\left(\alpha_{\varepsilon} u^{j}-\beta_{\varepsilon} u_{\varepsilon}\right)^{+}\right|^{2^{*}}-\left|\alpha_{\varepsilon} u^{j}\right|^{2^{*}}\right]\right| \leq 2^{*} \alpha_{\varepsilon}^{2^{*}-1} \beta_{\varepsilon} \int\left|u^{j}\right|^{2^{*}-1} u_{\varepsilon}\left|x_{i}\right|,
$$




$$
\left.\left|\int_{\Omega}\right|\left(\alpha_{\varepsilon} u^{j}-\beta_{\varepsilon} u_{\varepsilon}\right)^{+}\right|^{2^{*}}-\left.\int\left|\alpha_{\varepsilon} u^{j}\right|^{2^{*}}\left|\leq 2^{*} \alpha_{\varepsilon}^{2^{*}-1} \beta_{\varepsilon} \int\right| u^{j}\right|^{2^{*}-1} u_{\varepsilon} .
$$

From (3.15) and (3.16) we have

$$
\begin{aligned}
& g^{i}\left(\left(\alpha_{\varepsilon} u^{j}-\beta_{\varepsilon} u_{\varepsilon}\right)^{+}\right) \leq \frac{\int x_{i}\left|\alpha_{\varepsilon} u^{j}\right|^{2^{*}}+2^{*} 2^{2^{*}} \int\left|u^{j}\right|^{2^{*}-1} u_{\varepsilon}\left|x_{i}\right|}{\int\left|\alpha_{\varepsilon} u^{j}\right|^{2^{*}}-2^{*} 2^{2^{*}} \int\left|u^{j}\right|^{2^{*}-1} u_{\varepsilon}}, \\
& g^{i}\left(\left(\alpha_{\varepsilon} u^{j}-\beta_{\varepsilon} u_{\varepsilon}\right)^{+}\right) \geq \frac{\int x_{i}\left|\alpha_{\varepsilon} u^{j}\right|^{2^{*}}-2^{*} 2^{2^{*}} \int\left|u^{j}\right|^{2^{*}-1} u_{\varepsilon}\left|x_{i}\right|}{\int\left|\alpha_{\varepsilon} u^{j}\right|^{2^{*}}+2^{*} 2^{2^{*}} \int\left|u^{j}\right|^{2^{*}-1} u_{\varepsilon}} .
\end{aligned}
$$

Using (3.17) and the estimate

$$
\int_{\Omega}\left|u^{j}\right|^{2^{*}-1} u_{\varepsilon} \leq\left\|u^{j}\right\|_{L^{\infty}(\Omega)}^{2^{*}-1} \int_{\Omega} u_{\varepsilon} \leq C \varepsilon^{(N-2) / 2}
$$

we deduce that

$$
\begin{aligned}
g^{i}\left(u^{j}\right)-C_{1} \varepsilon^{(N-2) / 2} & =\frac{\int x_{i}\left|u^{j}\right|^{2^{*}}-C \varepsilon^{(N-2) / 2}}{\int\left|u^{j}\right|^{2^{*}}+C \varepsilon^{(N-2) / 2}} \\
& \leq g^{i}\left(\left(\alpha_{\varepsilon} u^{j}-\beta_{\varepsilon} u_{\varepsilon}\right)^{+}\right) \\
& \leq \frac{\int x_{i}\left|u^{j}\right|^{2^{*}}+C \varepsilon^{(N-2) / 2}}{\int\left|u^{j}\right|^{2^{*}}-C \varepsilon^{(N-2) / 2}} \\
& =g^{i}\left(u^{j}\right)+C_{2} \varepsilon^{(N-2) / 2}
\end{aligned}
$$

for some positive constants $C_{1}, C_{2}$, independent of $\varepsilon$, which implies, since $u^{j} \in$ $O_{\lambda}^{j}$ and $O_{\lambda}^{j}$ is open subset, that $g\left(\left(\alpha_{\varepsilon} u^{j}-\beta_{\varepsilon} u_{\varepsilon}\right)^{+}\right) \in B_{\ell}\left(a^{j}\right)$. The proof for $g\left(\left(\alpha_{\varepsilon} u^{j}-\beta_{\varepsilon} u_{\varepsilon}\right)^{-}\right) \in B_{\ell}\left(a^{j}\right)$ is similar. This completes the proof of (i).

Proof of (ii).

$$
I_{\lambda}\left(\alpha u^{j}-\beta u_{\varepsilon}\right)
$$




$$
\begin{aligned}
=I_{\lambda}\left(\alpha u^{j}\right)+ & \frac{\beta^{2}}{2} \int\left(\left|\nabla u_{\varepsilon}\right|^{2}-\lambda u_{\varepsilon}^{2}\right) \\
& -\alpha \beta \int Q(x)\left(u^{j}\right)^{2^{*}-1} u_{\varepsilon}-\frac{1}{2^{*}} \int Q(x)\left[\left|\alpha u^{j}-\beta u_{\varepsilon}\right|^{2^{*}}-\left(\alpha u^{j}\right)^{2^{*}}\right] \\
=I_{\lambda}\left(\alpha u^{j}\right)+ & \frac{\beta^{2}}{2} \int\left|\nabla u_{\varepsilon}\right|^{2}-\frac{1}{2^{*}} \int Q_{M}\left|\beta u_{\varepsilon}\right|^{2^{*}} \\
& -\lambda \frac{\beta^{2}}{2} \int u_{\varepsilon}^{2}+\frac{1}{2^{*}} \int\left|Q_{M}-Q(x)\right|\left|\beta u_{\varepsilon}\right|^{2^{*}} \\
& -\frac{1}{2^{*}} \int Q(x)\left[\left|\alpha u^{j}-\beta u_{\varepsilon}\right|^{2^{*}}-\left(\alpha u^{j}\right)^{2^{*}}-\left(\beta u_{\varepsilon}\right)^{2^{*}}+2^{*} \alpha \beta\left(u^{j}\right)^{2^{*}-1} u_{\varepsilon}\right] .
\end{aligned}
$$

Using the estimates in (2.7), (2.12) we also have $0 \leq \int\left(Q_{M}-Q(x)\left|\beta u_{\varepsilon}\right|^{2^{*}}=\right.$ $o\left(\varepsilon^{2}\right)$,

$$
\begin{aligned}
& \left|\int\right| \alpha u^{j}-\left.\beta u_{\varepsilon}\right|^{2^{*}}-\left(\alpha u^{j}\right)^{2^{*}}-\left(\beta u_{\varepsilon}\right)^{2^{*}}+2^{*} \alpha \beta\left(u^{j}\right)^{2^{*}-1} u_{\varepsilon} \mid \\
\leq & C\left(\int|\alpha|^{2^{*}-1} \beta\left|u^{j}\right|^{2^{*}-1} u_{\varepsilon}+|\alpha||\beta|^{2^{*}-1} u^{j} u_{\varepsilon}^{2^{*}-1}\right) \\
\leq & C_{1}\left(\int u_{\varepsilon}^{2^{*}-1}+u_{\varepsilon}\right) \\
= & O\left(\varepsilon^{(N-2) / 2}\right) .
\end{aligned}
$$

Therefore,

$$
\begin{array}{r}
\sup _{\alpha, \beta \in[1 / 2,2]} I_{\lambda}\left(\alpha u^{j}-\beta u_{\varepsilon}\right) \leq m_{\lambda}^{j}+\sup _{\beta \geq 0}\left[\frac{\beta^{2}}{2} \int\left(\left|\nabla u_{\varepsilon}\right|^{2}-\lambda u_{\varepsilon}^{2}\right)-\frac{\beta^{2^{*}}}{2^{*}} \int Q_{M} u_{\varepsilon}^{2^{*}}\right] \\
+o\left(\varepsilon^{2}\right)+O\left(\varepsilon^{(N-2) / 2}\right) .
\end{array}
$$

But we can show, as in Section 2, that

$$
\sup _{\beta \geq 0}\left[\frac{\beta^{2}}{2} \int\left(\left|\nabla u_{\varepsilon}\right|^{2}-\lambda u_{\varepsilon}^{2}\right)-\frac{\beta^{2^{*}}}{2^{*}} \int Q_{M} u_{\varepsilon}^{2^{*}}\right] \leq \frac{S^{N / 2}}{N Q_{M}^{(N-2) / 2}}-\lambda C \varepsilon^{2}+O\left(\varepsilon^{N-2}\right),
$$

for $N \geq 5$.

Therefore, we have

$$
\begin{aligned}
\sup _{\alpha, \beta \in[1 / 2,2]} I_{\lambda}\left(\alpha u^{j}-\beta u_{\varepsilon}\right) \leq m_{\lambda}^{j}+\frac{S^{N / 2}}{N Q_{M}^{(N-2) / 2}}-\lambda & C \varepsilon^{2}+O\left(\varepsilon^{N-2}\right) \\
& +o\left(\varepsilon^{2}\right)+O\left(\varepsilon^{(N-2) / 2}\right),
\end{aligned}
$$


which shows that, for $N \geq 7$, and $\varepsilon$ small,

$$
\sup _{\alpha, \beta \in[1 / 2,2]} I_{\lambda}\left(\alpha u^{j}-\beta u_{\varepsilon}\right)<m_{\lambda}^{j}+\frac{S^{N / 2}}{N Q_{M}^{(N-2) / 2}} .
$$

This completes the proof of (ii).

By Lemmas 2.2 and 3.1 it follows that there exists a $\lambda_{0} \in\left(0, \lambda_{1}\right)$ such that

$$
\bar{M}_{\lambda}^{j}>M_{\lambda}^{j}
$$

for all $\lambda \in\left(0, \lambda_{0}\right]$. A similar argument to the one used in Lemma 2.4 may be used to establish the following lemma.

Lemma 3.2. Assume condition $(Q)$ holds. Then there exists a $\lambda_{0} \in\left(0, \lambda_{1}\right)$ and a sequence $\left\{u_{n}^{j}\right\} \subset U_{\lambda}^{j}$, for each $j=1,2, \ldots, k$, satisfying

$$
\begin{aligned}
& I_{\lambda}\left(u_{n}^{j}\right) \rightarrow M_{\lambda}^{j}, \\
& I_{\lambda}^{\prime}\left(u_{n}^{j}\right) \rightarrow 0,
\end{aligned}
$$

as $n \rightarrow \infty$, for $\lambda \in\left(0, \lambda_{0}\right)$ and $j=1,2, \ldots, k$.

Proof of Theorem 1.2. From Lemma 2.3 and Lemma 3.2 we conclude that there exist a $\lambda_{0} \in\left(0, \lambda_{1}\right)$ and a sequence $\left\{u_{n}^{j}\right\} \subset U_{\lambda}^{j}$, for each $j=1,2, \ldots, k$, satisfying (3.18) and

$$
u_{n}^{j} \rightarrow u^{j} \quad \text { strongly in } H_{0}^{1}(\Omega)
$$

From (3.18) and (3.19) we have that $u^{j}, j=1, \ldots, k$, is a weak solution of (1.1). Since for $\lambda<\lambda_{1}$ there exists a positive constant $\delta$, independent of $u$, such that $\left\|u^{ \pm}\right\| \geq \delta>0$ for all $u^{ \pm} \in \Sigma_{\lambda},\left(u^{j}\right)^{ \pm} \not \equiv 0$. Finally, $u^{j}, j=1,2, \ldots, k$, are distinct nodal solutions since $g\left(u^{j}\right) \in B_{\ell}\left(a^{j}\right)$ and the $B_{\ell}\left(a^{j}\right)$ are disjoint. This completes the proof of Theorem 1.2.

\section{REFERENCES}

[1] F. V. Atkinson, H. Brezis \& L.A. Peletier, Nodal solutions of elliptic equations with critical Sobolev exponents, J. Differential Equations 85 (1990), 151-170.

[2] A. BAHRI \& J. M. CoRON, On a nonlinear elliptic equation involving the Sobolev exponent the effect of the topology of the domain, Comm. Pure Appl. Math. 41 (1988), $253-294$.

[3] V. Benci \& G. Cerami, The effect of the domain topology on the number of positive solutions of nonlinear elliptic problems, Arch. Rat. Mech. Anal. 11 (1971), 79-93. 
[4] H. BREZIS, Elliptic equations with limiting Sobolev exponents. The impact of topology, Comm. Pure Appl. Math. 39 (1986), 517-539.

[5] H. BREzIS \& E. LIEB, A relation between pointwise convergence of functions and convergence of functionals, Proc. Amer. Math. Soc. 88 (1988), 486-490.

[6] H. Brezis \& L. NiREnBerg, Positive solutions of nonlinear elliptic equations involving critical Sobolev exponents, Comm. Pure Appl. Math. 36 (1983), 437-477.

[7] G. Cerami 8 D. PASSASEO, Existence and multiplicity of positive solutions for nonlinear elliptic problems in exterior domains with rich topology, Nonlinear Analysis $\mathbf{1 8}$ (1992), 109-119.

[8] G. Cerami, S. Solimini \& M. Struwe, Some existence results for superlinear elliptic boundary value problems involving critical exponents, J. Funct. Anal. 69 (1986), 289-306.

[9] J. M. Coron, Topologie et cas limite des injections de Sobolev, C. R. Acad. Sci. Paris Sér I. Math. 299 (1984), 209-212.

[10] E. N. DANCER, A note on an equation with critical exponent, Bull. London Math. Soc. 20 (1988), 600-602.

[11] W. Ding, Positive solutions of $\Delta u+u^{(N+2) /(N-2)}=0$ on contractible domains, (Preprint).

[12] I. Ekeland, On the variational principle, J. Math. Anal. Appl. 17 (1974), 324-353.

[13] J. F. ESCOBAR, Positive solutions for some semilinear elliptic equations with critical Sobolev exponents, Comm. Pure. Appl. Math. 40 (1987), 623-657.

[14] J. KAZDAN 8 F. WARNER, Remarks on some quasilinear elliptic equations, Comm. Pure Appl. Math. 28 (1975), 567-597.

[15] P. L. LiONS, The concentration compactness principle in the calculus of variations: the limit case, Rev. Mat. Iberoamericana 1 (1985), 145-201; 45-121.

[16] M. V. MARChi \& F. PACELla, On the existence of nodal solutions of the equations $-\Delta u=|u|^{2^{*}-2} u$ with Dirichlet boundary conditions, Differential and Integral Equations 6 (1991), 849-862.

[17] C. Miranda, Un'osservazione sul teorema di Brouwer, Boll. Un. Mat. Ital. Ser. II 19 (1940), 5-7.

[18] D. PASSASEO, Multiplicity of positive solutions of nonlinear elliptic equations with critical exponents in some contractible domains, Manuscripta Math. 65 (1989), 147-166.

[19] S. I. Phozaev, Eigenfunctions of the equations $\Delta u+\lambda f(u)=0$, Sov. Math. Doklady 6 (1965), 1408-1411.

[20] P. N. SRIKANTh, Uniqueness of solutions of nonlinear Dirichlet problems, Differential and Integral equations 6 (1993), 663-670.

[21] G. TARANTEllo, On nonhomogeneous elliptic equations involving critical Sobolev exponent, Ann. Inst. H. Poincaré Anal. Non Linéaire 9 (1992), 281-304.

The first named author was partially supported by the National Sciences Foundation of China and the C.G. project of the Science and Technology Committee of Wuhan. The second named author was partially supported by the Australian National Research Council.

\section{DAOMIN CAO}

Wuhan Institute of Mathematical Sciences

The Chinese Academy of Sciences

P.O. Box 71007

Wuhan 430061

The Peoples' Republic of China
Ezzat S. Noussair

School of Mathematics

University of New South Wales

Sydney 2052 NSW, Australia

Received: January 9th, 1995. 